\title{
ON VARIETIES OF LEFT DISTRIBUTIVE LEFT IDEMPOTENT GROUPOIDS
}

\author{
DAVID STANOVSKÝ
}

\begin{abstract}
We describe a part of the lattice of subvarieties of left distributive left idempotent groupoids (i.e. those satisfying the identities $x(y z) \approx(x y)(x z)$ and $(x x) y \approx x y$ ) modulo the lattice of subvarieties of left distributive idempotent groupoids. A free groupoid in a subvariety of LDLI groupoids satisfying an identity $x^{n} \approx x$ decomposes as the direct product of its largest idempotent factor and a cycle. Some properties of subdirectly ireducible LDLI groupoids are found.
\end{abstract}

We consider groupoids (i.e. sets equipped with a binary operation) satisfying the following two identities:

$$
\begin{gathered}
x(y z) \approx(x y)(x z), \\
(x x) y \approx x y .
\end{gathered}
$$

We call such groupoids left distributive left idempotent, shortly LDLI. A groupoid is called idempotent, if it satisfies the identity

$$
x x \approx x .
$$

Note that the well known class of left distributive left quasigroups (see e.g. [2], [7], [8]) satisfies left idempotency. Indeed, our results can be applied there.

The purpose of this note is to continue recent investigations of P. Jedlička [3] on LDLI groupoids. We apply his result to compute the lattice of subvarieties of LDLI groupoids satisfying an identity $x^{n+1} \approx x$ for some $n$, modulo the lattice of subvarieties of LDI groupoids (see Theorem 4). This generalizes a result of T. Kepka [4] who described in a similar way subvarieties of LD groupoids with $x(x y) \approx y$ (such groupoids are called left symmetric; they satisfy LI and $x^{3} \approx x$ ).

In Section 2 we show some properties of subdirectly irreducible LDLI groupoids and apply them to get some information about the structure of the lattice of subvarieties satisfying identities $x^{m+n} \approx x^{m}$.

We use rather standard terminology and notation, for an introduction to universal algebra see e.g. [1]. We need the following result of P. Jedlicka [3]. Let $G$ be an LDLI groupoid and let $i p_{G}$ be the smallest equivalence on $G$ containing $\{(a, a a): a \in G\}$. Then $i p_{G}$ is a congruence, $G / i p_{G}$ is idempotent and $i p_{G}$ is the smallest congruence such that the corresponding factor is idempotent. Moreover,

1991 Mathematics Subject Classification. 08B15, 08B20, 20 N02.

Key words and phrases. left distributivity, subdirectly irreducible, free groupoid, lattice of subvarieties.

While working on this paper the author was partially supported by the Grant Agency of the Czech Republic, grants \#201/02/0594 and \#201/02/0148, and by the Institutional grant MSM113200007. 
for any $(a, b) \in i p_{G}, a c=b c$ holds for every $c \in G$. Consequently, any block of $i p_{G}$ is a subalgebra of $G$ and it is term equivalent to a connected monounary algebra.

\section{VARIETIES SATISFYING $x^{n+1} \approx x$}

Let us define inductively $x^{1}=x$ and $x^{n}=x x^{n-1}$ for every $n>1$. (Note that other possible definitions of powers do not really make sense: one can check by induction that any term $t$ in one variable is LI-equivalent to the term $x^{n}$, where $n$ is the depth of the rightmost variable in $t$.) It is easy to prove that in LDLI groupoids the identities $x y \approx x^{n} y$ and $\left(x^{k}\right)^{l} \approx x^{k+l-1}$ hold.

We say that a variety $\mathcal{V}$ of groupoids has exponent $n$, if $n$ is the least positive integer such that the identity $x^{n+1} \approx x$ holds in $\mathcal{V}$. (Of course, such $n$ does not necessarily exist, however, many important varieties, for instance left $n$-symmetric left distributive groupoids (see e.g. [8]), have finite exponent.)

Let $C_{n}$ denote the groupoid on the set $\{0, \ldots, n-1\}$ with the operation $a b=b+1$ $\bmod n$. Clearly, $C_{n}$ are LDLI groupoids.

Lemma 1. Let $G$ be an LDLI groupoid with $x^{n+1} \approx x$. Then

(1) every block of $i p_{G}$ is isomorphic to $C_{k}$ for some $k \mid n$;

(2) $C_{n}$ is a homomorphic image of $G$, if and only if $G$ is isomorphic to the direct product $C_{n} \times\left(G / i p_{G}\right)$.

Proof. (1) is easy. For (2), choose a projection $g: G \rightarrow C_{n}$ and put $f(x)=$ $\left(g(x), x / i p_{G}\right)$. Then $f: G \rightarrow C_{n} \times\left(G / i p_{G}\right)$ is a homomorphism. Since there is a homomorphism $C_{k} \rightarrow C_{l}$ iff $l \mid k$, every block of $i p_{G}$ is isomorphic to $C_{n}$ ( $g$ restricted to a block of $i p_{G}$ is a homomorphism). Hence $g$ is bijective on every block of $i p_{G}$, because rotations are the only endomorphisms of $C_{n}$, and thus $f$ is an isomorphism. The other implication is clear.

Lemma 2. Let $\mathcal{V}$ be a subvariety of LDLI groupoids and assume $\mathcal{V}$ has exponent $n$. Then $C_{k} \in \mathcal{V}$, iff $k \mid n$.

Proof. If $k$ does not divide $n$, then $C_{k}$ does not satisfy $x^{n+1} \approx x$. On the other hand, if $\mathcal{V}$ has exponent $n$, then, according to Lemma 1, ip-blocks of elements of $\mathcal{V}$ are $C_{k}$ with $k \mid n$ (indeed, ip-blocks are subgroupoids). Let $k_{0}$ be the greatest $k$ such that $C_{k} \in \mathcal{V}$. If $k_{0}<n$, then $\mathcal{V}$ satisfies $x^{k_{0}+1} \approx x$, a contradiction with minimality of $n$. Hence $C_{n} \in \mathcal{V}$ and thus $C_{k} \in \mathcal{V}$ for all $k \mid n$, because they are homomorphic images of $C_{n}$.

Let $\mathbf{F}_{\mathcal{V}}(X)$ denote the free groupoid over $X$ in a variety $\mathcal{V}$. Let $\mathcal{I}$ denote the variety of idempotent groupoids.

Theorem 3. Let $\mathcal{V}$ be a subvariety of LDLI groupoids and assume $\mathcal{V}$ has exponent n. Then $\mathbf{F}_{\mathcal{V}}(X)$ is isomorphic to $C_{n} \times \mathbf{F}_{\mathcal{V} \cap \mathcal{I}}(X)$. Consequently, the variety $\mathcal{V}$ is generated by $(\mathcal{V} \cap \mathcal{I}) \cup\left\{C_{n}\right\}$.

Proof. Since $C_{n} \in \mathcal{V}$, it is a homomorphic image of $\mathbf{F}_{\mathcal{V}}(X)$. Hence, by Lemma 1, $\mathbf{F}_{\mathcal{V}}(X) \simeq C_{n} \times H$, where $H=\mathbf{F}_{\mathcal{V}}(X) / i p_{\mathbf{F}_{\mathcal{V}}(X)}$. It is easy to see that $H \simeq \mathbf{F}_{\mathcal{V} \cap \mathcal{I}}(X)$, because $i p$ is the smallest idempotent congruence.

A right zero band is a groupoid satisfying the identity $x y \approx y$. It is well known that the variety $\mathcal{R} \mathcal{Z} \mathcal{B}$ of right zero bands is minimal (i.e. it is generated by each of its elements). 
Theorem 4. Let $L$ denote the lattice of subvarieties of LDI groupoids, $K$ its sublattice of varieties containing $\mathcal{R Z B}$ and $N$ the lattice of positive integer divisors of $n$. The lattice of subvarieties of the variety of LDLI groupoids satisfying $x^{n+1} \approx x$ is isomorphic to the lattice $(L \times\{1\}) \cup(K \times(N \backslash\{1\}))$ (regarded as a subposet of $L \times N)$, sending a variety $\mathcal{V}$ of exponent $m$ to the pair $\Phi(\mathcal{V})=(\mathcal{V} \cap \mathcal{I}, m)$.

Proof. First, we check that the mapping $\Phi$ is well-defined: the exponent $m$ of a subvariety $\mathcal{V}$ is clearly a divisor of $n$ and since $\mathcal{V}$ contains $C_{m}$, it contains a right zero band $\left(C_{m} \times C_{m}\right) / i p$ and thus it contains the whole variety $\mathcal{R} \mathcal{Z} \mathcal{B}$, because it is minimal. Next, $\Phi$ is injective: if $\mathcal{V}_{1}$ and $\mathcal{V}_{2}$ are distinct varieties of exponent $m$, then $\mathcal{V}_{1} \cap \mathcal{I}$ and $\mathcal{V}_{2} \cap \mathcal{I}$ are distinct, because $\mathcal{V}_{i}$ is generated by $\left(\mathcal{V}_{i} \cap \mathcal{I}\right) \cup\left\{C_{m}\right\}$, $i=1,2$. The mapping $\Phi$ is onto, a pair $(\mathcal{W}, m)$ is the image of the variety generated by $\mathcal{W} \cup\left\{C_{m}\right\}$. Indeed, let $G$ be an idempotent groupoid in the variety generated by $\mathcal{W} \cup\left\{C_{m}\right\}$ and we show that $G \in \mathcal{W}$. The case $m=1$ is trivial, so let $m>1$. By Birkhoff's HSP theorem, there are $H \in \mathcal{W}, K \leq H \times C_{m}^{k}$ (for some $k$ ) and an onto homomorphism $\varphi: K \rightarrow G$. Since $i p_{K}$ is the smallest idempotent congruence and $G$ is idempotent, there is an onto homomorphism $\psi: K / i p_{K} \rightarrow G$. Further, $K / i p_{K} \leq\left(K \times C_{m}^{k}\right) / i p \simeq K \times\left(C_{m}^{k} / i p\right)$. However, $C_{m}^{k} / i p$ is a right zero band and thus it is in $\mathcal{W}$. Consequently, $G$ is a homomorphic image of a subgroupoid of a groupoid from $\mathcal{W}$, thus it is in $\mathcal{W}$. Finally, $\Phi$ clearly preserves the order and it follows from Theorem 3 that also $\Phi^{-1}$ preserves the order. Consequently, $\Phi$ is a lattice isomorphism.

Example. B. Roszkowska proved in [6] that the lattice of subvarieties of left symmetric medial idempotent (LSMI) groupoids (those where the identities $x(x y) \approx y$, $x y \cdot u v \approx x u \cdot y v$ and $x x \approx x$ hold) is isomorphic to the lattice of positive integers ordered by divisibility with a top element added. A number $n$ corresponds to the variety based on $w_{n}(x, y) \approx y$ (relatively to LSMI), where

$$
w_{n}(x, y)=\underbrace{x(y(x(y(\ldots))))}_{n} .
$$

Note that right zero bands satisfy $w_{n}(x, y) \approx y$ iff $n$ is even. Thus, using Theorem 4 , it is easy to describe bases of all proper subvarieties of left symmetric left distributive medial groupoids (relatively to LSLDM):

(1) $x x \approx x$

(2) $w_{n}(x, y) \approx y$ and $x x \approx x$, for every $n$

(3) $w_{n}(x, y) \approx y$, for every $n$ even.

(Note that mediality and idempotency imply left distributivity, however, nonidempotent medial groupoids are not necessarily left distributive.)

Example. J. Płonka [5] investigated idempotent groupoids satisfying

$$
\underbrace{x(x(\ldots(x)}_{n} y)) \approx y, \quad x(y z) \approx y(x z) \quad \text { and } \quad x z \approx(y x) z .
$$

He called them $n$-cyclic groupoids. It is easy to see that they are LDLI and that 1-cyclic groupoids are precisely right zero bands. Płonka proved that the only non-trivial subvarieties of $n$-cyclic groupoids are $m$-cyclic groupoids for $m \mid n$. One can thus use Theorem 4 to describe the subvarieties of non-idempotent $n$-cyclic groupoids. Every non-trivial one is generated by idempotent $m$-cyclic groupoids 
and the groupoid $C_{k}$, for some divisors $m, k$ of $n$; hence there are exactly $q^{2}+1$ such subvarieties, where $q$ is the number of divisors of $n$.

\section{VARIETIES SATISFYING $x^{m+n} \approx x^{m}$}

Theorems 3 and 4 cannot be generalized to varieties satisfying an identity $x^{m+n} \approx$ $x^{m}$ for $m>1$. For instance, in an LDLI groupoid $G$ with $x^{3} \approx x^{2}$, every $i p_{G}$-block is a constant groupoid, i.e. $a b=c d$ for all $i p_{G^{-}}$congruent elements $a, b, c, d$ (the corresponding unary algebra is a loop with several "tails" of length 1). However, this variety is not generated by LDI and constant groupoids. Indeed, both LDI and constant groupoids satisfy the identity $x y \approx x(y y)$, while the groupoid

\begin{tabular}{c|cccc} 
& $a$ & $b$ & $c$ & $d$ \\
\hline$a, b$ & $b$ & $b$ & $c$ & $d$ \\
$c, d$ & $a$ & $b$ & $d$ & $d$
\end{tabular}

does not, though it is LDLI with $x^{3} \approx x^{2}$. We thus present a weaker result for such varieties.

For a variety $\mathcal{V}$, let $\mathcal{V}_{m, n}$ denote the subvariety of $\mathcal{V}$ based (relatively to $\mathcal{V}$ ) by the identity $x^{m+n} \approx x^{m}$.

It is well known that a groupoid $G$ is subdirectly irreducible, iff it possesses the smallest non-trivial congruence, and that any variety is generated by its subdirectly irreducible members.

Lemma 5. Let $G$ be a subdirectly irreducible LDLI groupoid. If $C_{n}$ is a subalgebra of $G$ for some $n \geq 2$, then $G$ contains no fork (i.e. elements $a \neq b$ with $a^{2}=b^{2}$ ) and, vice versa, if $G$ contains a fork, then $C_{n}$ is not a subalgebra of $G$, for any $n \geq 2$.

Proof. Put $\alpha=\left\{(a, b) \in G \times G: a^{2}=b^{2}\right\}$. It is clear that $\alpha$ is an equivalence, which glues each fork. It is a congruence, because whenever $a^{2}=b^{2}$, we get $a t=a^{2} t=b^{2} t=b t$ and $(t a)^{2}=t a^{2}=t b^{2}=(t b)^{2}$.

Put $(a, b) \in \beta$ iff there are $k, l$ such that $a^{k}=b$ and $b^{l}=a$. By a similar argument, it is easy to see that $\beta$ is a congruence, which glues each circle.

Clearly, $\alpha \cap \beta=i d_{G}$, hence either $\alpha=i d_{G}$ or $\beta=i d_{G}$ or both, and thus either $G$ contains no fork, or no circle with two or more elements, or both.

Remark. One can prove that a subdirectly irreducible LDLI groupoid $G$ either contains a fork, or there is a prime $p$ and a natural number $k$ such that all $i p_{G}$-blocks are circles of length either 1 , or $p^{k}$ (this is proven in [8] for LD left quasigroups, however, it is sufficient to assume in the proof LDLI only). On the other hand, there seems to be no uniformity in the former case. In the following example the $i p$-blocks have different length of tails and, moreover, one contains a 'pure fork' (such that $b \neq b^{2}=c^{2} \neq c$ ), while the other don't. The smallest non-trivial congruence has the only non-trivial block $\left\{a^{2}, a^{3}\right\}$.

\begin{tabular}{c|ccccccc} 
& $a$ & $a^{2}$ & $a^{3}$ & $b$ & $c$ & $b^{2}$ & $d$ \\
\hline$a, a^{2}, a^{3}$ & $a^{2}$ & $a^{3}$ & $a^{3}$ & $a^{2}$ & $a^{2}$ & $a^{3}$ & $d$ \\
$b, c, b^{2}=c^{2}$ & $a^{3}$ & $a^{3}$ & $a^{3}$ & $b^{2}$ & $b^{2}$ & $b^{2}$ & $d$ \\
$d$ & $a^{3}$ & $a^{3}$ & $a^{3}$ & $a^{2}$ & $a^{3}$ & $a^{3}$ & $d$
\end{tabular}

Lemma 6. Let $\mathcal{V}$ be a subvariety of LDLI groupoids and $m, n$ be positive integers. Then $\mathcal{V}_{m, n}$ is the join of the varieties $\mathcal{V}_{m, 1}$ and $\mathcal{V}_{1, n}$. 
Proof. Let $G$ be an LDLI groupoid with $x^{m+n} \approx x^{m}$. Then $i p_{G^{-}}$-blocks consist of a circle of length $k$, where $k \mid n$, and possibly some "tails" of length at most $m$ (precisely, for any element $a$ out of the circle, $a^{m}$ lies on the circle). Now, assume that $G$ is subdirectly irreducible. It follows from the previous lemma that either all the circles are of length one, or there are no tails (because whenever a tail joins a circle, there is a fork). Hence, $G$ satisfies either $x^{m+1} \approx x^{m}$ (tails of length at most $m$ only), or $x^{n+1} \approx x$ (circles only). Now, the claim follows from the fact that any variety is generated by its subdirectly irreducible members.

Theorem 7. Let $\mathcal{V}$ be a subvariety of LDLI groupoids and let $k, l, m, n$ be positive integers. Then

$$
\mathcal{V}_{k, l} \vee \mathcal{V}_{m, n}=\mathcal{V}_{\max (k, m), \operatorname{LCM}(l, n)}
$$

and

$$
\mathcal{V}_{k, l} \wedge \mathcal{V}_{m, n}=\mathcal{V}_{\min (k, m), \mathrm{GCD}(l, n)} .
$$

Proof. For the first equality, use the previous lemma and compute $\mathcal{V}_{k, l} \vee \mathcal{V}_{m, n}=$ $\mathcal{V}_{k, 1} \vee \mathcal{V}_{m, 1} \vee \mathcal{V}_{1, l} \vee \mathcal{V}_{1, n}=\mathcal{V}_{\max (k, m), 1} \vee \mathcal{V}_{1, \operatorname{LCM}(l, n)}=\mathcal{V}_{\max (k, m), \operatorname{LCM}(l, n)}$. The second claim is rather clear.

Let $\mathcal{V}$ be a variety of LDLI groupoids such that it satisfies no identity $x^{m+n} \approx x^{m}$. We show that the mapping $(m, n) \mapsto \mathcal{V}_{m, n}$ is injective on $\mathbb{N} \times \mathbb{N}$. The identity $x z \approx y z$ implies an identity $t \approx s$ iff the depth of the rightmost variable in $t$ equals to the depth of the rightmost variable in $s$ and the two variables are identical. Indeed, all identities of $\mathcal{V}$ have the latter property - otherwise $t(x, \ldots, x) \approx s(x, \ldots, x)$ were a non-trivial identity in one variable. Hence $\mathcal{V}$ contains all groupoids with $x z \approx y z$, i.e., in fact, unary algebras. It is easy to see that for any $m_{1}, n_{1}$ and $m_{2}, n_{2}$ with $\left(m_{1}, n_{1}\right) \neq\left(m_{2}, n_{2}\right)$ there is a unary algebra such that it satisfies exactly one of the identities $x^{m_{1}+n_{1}} \approx x^{m_{1}}, x^{m_{2}+n_{2}} \approx x^{m_{2}}$. Hence, $\mathcal{V}_{m_{1}, n_{1}} \neq \mathcal{V}_{m_{2}, n_{2}}$. Moreover, $\mathcal{V}_{1,1}$ contains $\mathcal{R Z B}$.

However, not every subvariety of LDLI groupoids is equal to $\mathcal{V}_{m, n}$ for some $\mathcal{V}, m, n$. For instance, consider the variety $\mathcal{C}$ of constant groupoids (satisfying the identity $x y \approx u v$ ); clearly, $x^{3} \approx x^{2}$ holds in $\mathcal{C}$. Suppose there is a variety $\mathcal{V}$ such that $\mathcal{V}_{2,1}=\mathcal{C}$. Then $\mathcal{V}_{1,1}$ is a non-trivial idempotent subvariety of $\mathcal{C}$. However, there is no non-trivial idempotent groupoid in $\mathcal{C}$, a contradiction.

Problem. As shown above, the claim of Theorem 3 does not work for varieties without exponent. Particularly interesting case is the following: describe the structure of free LDLI groupoids modulo free LDI ones.

\section{REFERENCES}

[1] S. Burris, H.P. Sankappanavar, A course in universal algebra, GTM 78, Springer, 1981.

[2] R. Fenn, C. Rourke, Racks and links in codimension two, J. Knot Theory Ramifications 1 (1992), 343-406.

[3] P. Jedlička, On left distributive left idempotent groupoids, Comment. Math. Univ. Carolinae, to appear.

[4] T. Kepka, Non-idempotent left symmetric left distributive groupoids, Comment. Math. Univ. Carolinae 35/1 (1994), 181-186.

[5] J. Płonka, On k-cyclic groupoids, Mat. Japonica 30 (1985), 371-382.

[6] B. Roszkowska, The lattice of varieties of symmetric idempotent entropic groupoids, Demonstratio Math. 20 (1987), 259-275.

[7] H. Ryder, The congruence structure of racks, Commun. Alg. 23 (1995), 4971-4989. 
[8] D. Stanovský, Left distributive left quasigroups, PhD Thesis, Charles University in Prague, 2004. Available at http://www.karlin.mff.cuni.cz/〜 stanovsk/math/disert.pdf

David Stanovský, Charles University in Prague, Czech Republic

E-mail address: stanovsk@karlin.mff.cuni.cz 